\title{
Tailor-made PEG-DA-CuS nanoparticles enriched in tumor with the aid of retro Diels-Alder reaction triggered by their intrinsic photothermal property
}

This article was published in the following Dove Press journal: International Journal of Nanomedicine

\author{
Jie Sheng ${ }^{1,2}$ \\ Beibei Ma' \\ Qian Yang' \\ Chao Zhang ${ }^{2}$ \\ Zhongying Jiang ${ }^{1,2}$ \\ Entomack Borrathybay ${ }^{3}$ \\ 'College of Electronic and \\ Information Engineering, Yili Normal \\ University, Micro-nano Electric \\ Sensing Technology and Bionic \\ Devices Key Laboratory, Yining \\ 835000 , China; ${ }^{2}$ Physics School of \\ Nanjing University, Laboratory of \\ Solid State Microstructures, Nanjing \\ 210093 , China; ${ }^{3}$ College of Biology \\ and Geography Sciences, Yili Normal \\ University, Yining 835000, Xinjiang, \\ China
}

Introduction: In recent years, near-infrared laser-induced photothermal therapy is being considered as a promising approach to kill tumors owing to its noninvasive nature and excellent antitumor efficiency. However, the lack of ideal photothermal agents hinders further development of this technology.

Materials and methods: Aiming at solving this long-standing obstacle, we report here about the polyethylene glycol (PEG)-DA modified copper sulfide (CuS) nanoparticles (NPs) (PEGDA-CuS NPs), a kind of semiconductor photothermal agents that show excellent photothermal stability and high heat conversion efficiency.

Results and discussion: Owing to the surrounding PEG, the water solubility of CuS NPs was significantly improved when circulating in blood in the body. When the NPs reached the tumors and were irradiated by a 1,064 $\mathrm{nm}$ laser $\left(1 \mathrm{~W} / \mathrm{cm}^{2}, 10\right.$ minutes $)$, the local temperature increased above $90^{\circ} \mathrm{C}$, triggering the retro Diels-Alder reaction. After the release of PEG chain, $\mathrm{CuS}$ NPs soon formed aggregates and enriched the tumor via the enhanced permeability and retention effect, promoting the efficacy of photothermal therapy.

Conclusion: Therefore, we believe PEG-DA-CuS NPs are able to serve as a kind of cytotoxic and efficient photothermal agent to kill cancer.

Keywords: CuS, PEG, retro Diels-Alder reaction, excellent photothermal stability, high heat conversion efficiency, aggregation in tumor

\section{Introduction}

With the accumulating fundamental study of tumor, we realized that tumor is not only a mass formed by malignant cells, but also a highly heterogeneous multi-cell aggregation. Tumor microenvironment plays a decisive role in the occurrence, development, and treatment of tumor tissues. ${ }^{1,2}$ The terrible extracellular environment including metabolic stress, lack of oxygen, nutrients and growth factors, lack of blood supply insufficiency, and antitumor treatment ensures tumor cells the ability to resist tumor apoptosis, thus greatly improving the ability to survive adversity.,4 Traditional cancer treatments include surgery, radiation, and chemotherapy. However, they all have obvious flaws. The surgery cannot remove the small lesions that the human eye cannot see. Chemotherapy often causes tumor cell resistance and tumor recurrence after treatment. ${ }^{5}$ Radiotherapy faces the risk of carcinogenesis in normal tissues. ${ }^{6}$ Therefore, it is of great therapeutic and fundamental significance to develop new strategies for tumor treatment.

Near-infrared (NIR) laser-induced photothermal therapy (PTT) has attracted significant attention due to the noninvasive nature and safe biocompatibility of NIR laser 
irradiation in normal tissues. ${ }^{7}$ In the development of PTT, obtaining ideal photothermal agents is the key step, since they can convert absorbed optical energy into heat, which enables the ablation of tumors while preventing the surrounding tissues from being affected. ${ }^{8-18}$ So far, a variety of functional nanoparticles (NPs) have been examined, including polymer NPs, noble metal nanomaterials, carbon-based nanomaterials, and semiconductor nanomaterials, due to their strong absorbency under NIR. ${ }^{19-26}$ Among them, semiconductor copper sulfide $(\mathrm{CuS}) \mathrm{NPs}$ possess excellent physical properties and high heat conversion efficiency, which triggers our interest in exploring their clinical applications in photothermal ablation of tumors. Compared with noble metal nanomaterials such as golden nanorods, CuS NPs exhibit higher heat tolerance. Club-shaped golden nanorods are easily transformed to spherical shape at a high temperature. ${ }^{27}$ On the contrary, uniformly spherical $\mathrm{CuS}$ NPs have a stable conformation, which does not change after the hyperthermia process, ensuring their high heat conversion efficiency after repeated laser irradiation. In addition, their absorption peaks are positioned at 1,060 nm, longer than that of golden nanorods, which means they can efficiently absorb laser of longer wavelength and lower energy, causing smaller side effects to normal tissues. Unfortunately, considering their fast metabolic rate, CuS NPs can hardly accumulate into tumor cells, which is bound to limit the efficacy of PTT. In order to overcome this obstacle, various approaches have been explored. Meng's group used thermosensitive $\mathrm{MEO}_{2} \mathrm{MA}$ nanogel as a nanocarrier to deliver CuS NPs. ${ }^{28}$ Zhang et al's article introduced an activatable Cy5.5-hyaluronic acid nanoparticles/CuS (HANPC) nanocomposite to achieve optical/photoacoustic image-guided PTT. ${ }^{29}$ However, these methodologies capable of loading and delivering CuS NPs all relied on a complicated vehicle and an immediate outside stimulus to obtain a good delivery outcome. As such, these systems failed in taking advantage of the excellent photothermal qualities of CuS NPs.

Here, we successfully synthesized polyethylene glycol (PEG)-linked Diels-Alder cycloadducts-modified CuS NPs (PEG-DA-CuS NPs). The Diels-Alder reaction is a reversible reaction, and the forward reaction proceeds at $60^{\circ} \mathrm{C}$ while the backward reaction proceeds at $90^{\circ} \mathrm{C} .{ }^{30}$ In the forward reaction process, the water solubility of $\mathrm{CuS}$ NPs was improved thanks to the presence of PEG on their surfaces. When the PEG-DA-modified CuS NPs were injected into the body, they were stable and had prolonged circulation life in the blood. After they reached the tumor area, we used $1,064 \mathrm{~nm}$ laser to irradiate the lesion to obtain a local high temperature of $>90^{\circ} \mathrm{C}$. Then, the backward reaction (retro Diels-Alder reaction) happened, releasing PEG chains from the CuS NPs. After laser irradiation, the aggregation and enrichment of CuS NPs in the tumor via enhanced permeability and retention effect were rigorously demonstrated by the in vivo biodistribution of CuS NPs. In vitro and in vivo tests showed that PTT rendered by the PEG-DA-CuS NPs under 1,064 nm laser irradiation maintained a wonderful effect on cancer treatment and effectively erased the tumor. The whole process is illustrated in Scheme 1. We believe these tailor-made PEG-DA-CuS NPs will open a new avenue for tumor treatment and throw light on their clinical applications in cancer.

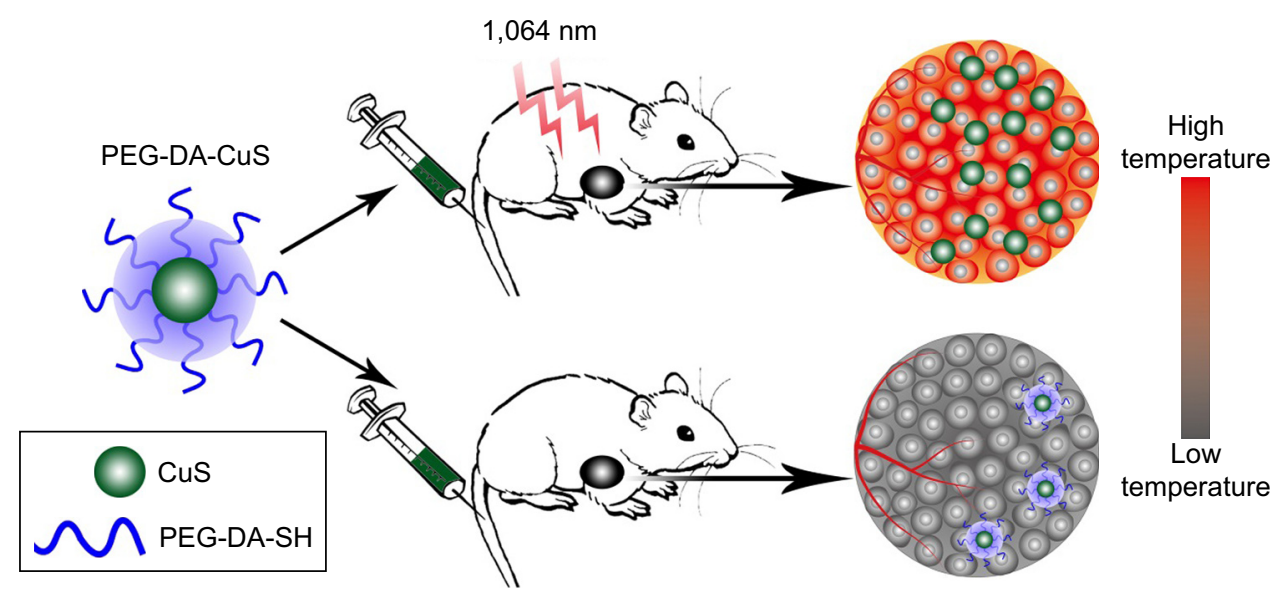

Scheme I When the PEG-DA-modified CuS nanoparticles were injected in the body, they were stable and had prolonged circulation life in the blood. After they reached the tumor area, I,064 nm laser was employed to irradiate the lesion to obtain a local high temperature of $>90^{\circ} \mathrm{C}$; then the backward reaction (retro Diels-Alder reaction) happened, releasing the PEG chains from the CuS nanoparticles. After laser irradiation, the aggregation and enrichment of CuS nanoparticles displayed a superior photothermal therapy.

Abbreviation: PEG, polyethylene glycol. 


\section{Materials and methods}

$\mathrm{CuCl}_{2} \cdot 2 \mathrm{H}_{2} \mathrm{O}$, sodium hydroxide $(\mathrm{NaOH})$, and thioacetamide were all purchased from Aladdin (Shanghai, China). m-PEGmaleimide (molecular weight 20,000 Da) was bought from J\&K Chemicals (Beijing, China). Calcein-acetoxymethyl (calcein-AM), dimethylformamide, tris (2-carboxyethyl) phosphine hydrochloride, ethidium homodimer-1 (EthD-1), furfuryl disulfide, and PBS ( $\mathrm{pH} \sim 7.4$ ) were all bought from Jinpan biotech (Nantong, China). Human umbilical vein endothelial cells (HUVECs) and human cervical carcinoma (HeLa) cell line were both ordered from Servicebio Company (Wuhan, China). Nude mice (female, 4-6 weeks) were bought from Comparative Medicine Center of Yangzhou University. Eight mice per cage were housed under controlled environmental conditions $\left(22^{\circ} \mathrm{C} \pm 3{ }^{\circ} \mathrm{C}, 45 \%-70 \%\right.$ relative humidity; 12-hour light/dark cycle). All animal experiments were approved by the Care Committee of Nanjing University (including guidelines for animal care \& use, and guidelines for euthanasia of mice, Protocol \#: 20170322-016).

\section{Preparation of PEG-DA-CuS NPs}

PEG-DA-CuS NPs were synthesized as illustrated in Scheme 2. First, PEG-DA-SH was prepared according to previously reported procedures. ${ }^{30}$ Then, an aqueous solution
(40 $\mathrm{mL})$ of the as-obtained PEG-DA-SH $(0.1 \mathrm{mmol})$ was mixed with an aqueous solution $(40 \mathrm{~mL})$ of $\mathrm{CuCl}_{2} \cdot 2 \mathrm{H}_{2} \mathrm{O}$ $(0.1 \mathrm{mmol})$ and made to react for 10 minutes. Afterward, an aqueous solution $(10 \mathrm{~mL})$ of $\mathrm{NaOH}(0.1 \mathrm{mmol})$ was added dropwise to adjust the solution $\mathrm{pH}$ to 9 . Then, the whole solution was transferred to a three-necked flask and an aqueous solution $(10 \mathrm{~mL})$ of thioacetamide $(0.1 \mathrm{mmol})$ was added. The solution was under constant stirring at $60^{\circ} \mathrm{C}$ for 1.5 hours, and the process was protected by $\mathrm{N}_{2}$ atmosphere.

\section{Characterization}

The morphologies of the obtained NPs were observed by transmission electron microscopy (TEM; JEOL, Tokyo, Japan), and the ultraviolet (UV)-Vis absorption spectra were recorded on a spectrophotometer (UV3100; Shimadzu Corporation, Kyoto, Japan). The size distribution was confirmed by dynamic light scattering method (BI-9000AT; Brookhaven, MS, USA). To study the photothermal effects, a 1,064 nm diode laser (LELS-1064-3000TFC-D; LaserGlow Technologies, Toronto, Canada) was used. The photothermal conversion efficiency ( $)$ was derived from the following fomula: ${ }^{10,27}$

$$
\eta(\%)=\frac{c m t}{w t} \times 100 \%=\frac{4.2 \times 10^{3} \times 1.43 \times 10^{-3} t}{1 \times 600} \times 100 \%
$$

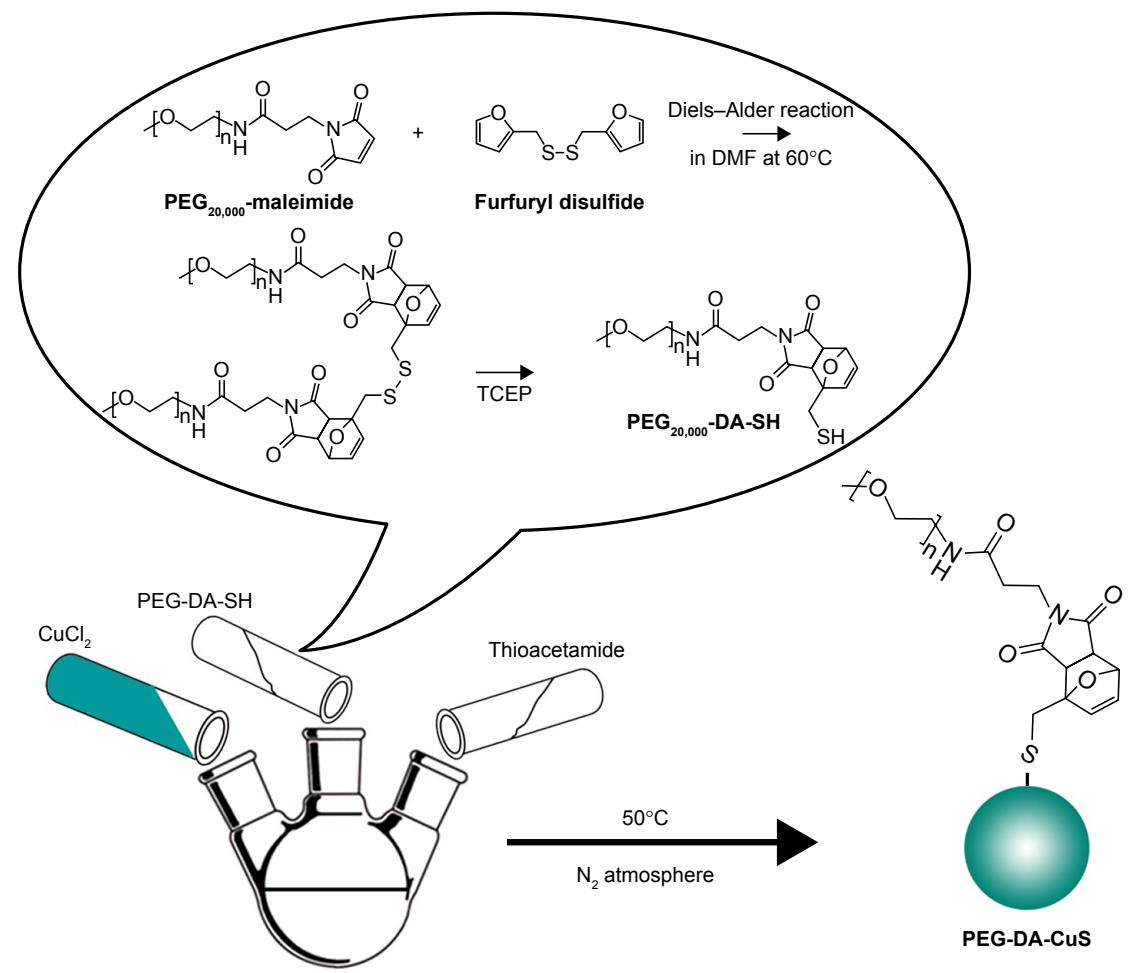

Scheme 2 Synthesis of PEG-DA-CuS NPs.

Abbreviations: DMF, dimethylformamide; NP, nanoparticle; PEG, polyethylene glycol; TCEP, tris (2-carboxyethyl) phosphine hydrochloride. 
where $w$ represents the laser power, $1 \mathrm{~W}$; m represents the mass of PEG-DA-CuS NP solution, $1.43 \times 10^{-3} \mathrm{~kg}$; $t$ represents the laser irradiation time, 600 seconds; c represents the specific heat capacity of PEG-DA-CuS NP solution, $4.2 \times 10^{3} \mathrm{~J} /(\mathrm{kg} \cdot \mathrm{K})$; and $\Delta$ t represents the temperature increase of PEG-DA-CuS NP solution. Release of PEG chains from PEG-DA-CuS NPs was quantified by the barium iodine method. ${ }^{30}$

\section{In vitro cytotoxicity assay}

The Annexin-V-fluorescein isothiocyanate/propidium iodide staining method was used to evaluate the cytotoxicity of PEG-DA-CuS NPs to HUVECs. At first, HUVECs were seeded into 96 -well plates $\left(1 \times 10^{4}\right.$ cells, $100 \mathrm{~mL}$ medium, per well) and incubated in $5 \% \mathrm{CO}_{2}$ atmosphere at $37^{\circ} \mathrm{C}$ for 1 day. Then, the cells were washed twice with PBS, followed by incubating them with PEG-DA-CuS NPs of various concentrations $(0.1,0.2,0.4$, and $1 \mathrm{mg} / \mathrm{mL})$. Afterward, the cells were cultured with fresh culture medium at $37^{\circ} \mathrm{C}$ for 4 hours. Finally, the cells were collected and resuspended in a binding buffer $(500 \mu \mathrm{L})$. Annexin V-fluorescein isothiocyanate and propidium iodide were added following the manufacturer's guidance. After incubation in darkness at room temperature for 20 minutes, samples were analyzed using flow cytometry (FACS verse; BD Biosciences, Franklin Lake, NJ, USA) and relevant FlowJo software.

\section{Biodistribution of PEG-DA-CuS NPs}

An aqueous solution (0.1 mL) of PEG-DA-CuS NPs (100 mg) was intravenously (iv) administered into the HeLa-xenograftbearing mice with the mice divided into two groups. One group received no laser irradiation, while the other group was irradiated by $1,064 \mathrm{~nm}$ laser 0.5 hour after the injection. The mice were sacrificed (six mice per time point) at $0.5,1,3,8,12$, and 24 hours after the injection. The tumor, liver, heart, spleen, kidney, lung, and blood were collected, weighed, completely lysed in aqua regia, and examined by an inductively coupled plasma-mass spectrometer (NexION 300 D; PerkinElmer Inc., Waltham, MA, USA).

\section{In vitro antitumor effect}

To assess the in vitro PTT rendered by PEG-DA-CuS NPs, HeLa cells were incubated in the confocal dish for a day at $37^{\circ} \mathrm{C}$. Then, the HeLa cells were cultured with PEG-DACuS NPs $(200 \mu \mathrm{g} / \mathrm{mL})$ and incubated for 5 hours. The, the media were discarded and the cells were washed with PBS thrice and exposed to $1,064 \mathrm{~nm}$ laser at a power of $1 \mathrm{~W} / \mathrm{cm}^{2}$ (10 minutes). The viability of HeLa cells posttreatment was indicated by staining the cells with calcein-AM or EthD-1 accordingly. Finally, laser scanning confocal microscopy was used to determine the cells' viability with and without formalin fixation.

\section{In vivo antitumor effect}

Mice bearing HeLa tumors in the right flank, with average tumor sizes of $500 \mathrm{~mm}^{3}$, were used to assess the in vivo antineoplastic efficiency. The mice were randomized into four different groups (iv injection of saline $[50 \mathrm{mg} / \mathrm{kg}$ ], iv injection of PEG-DA-CuS [50 mg/ $\mathrm{kg}$ ] alone, iv injection of saline $[50 \mathrm{mg} / \mathrm{kg}$ ] plus $1,064 \mathrm{~nm}$ laser, iv injection of PEG-DA-CuS [50 mg/kg] plus 1,064 nm laser). Tumor size was evaluated by monitoring and recording the volume and body weight. After the mice were sacrificed 12 days after the treatments, the tumors were collected, weighed, and fixed in a 10\% neutral buffered formalin solution after three washes with PBS. Immunohistochemistry for Ki67 staining tests and H\&E staining tests were also conducted by Yili biotechnology Company (Wu Han, China) and observed via fluorescence microscopy (IX71; Olympus Corporation, Tokyo, Japan).

\section{Pathology analysis}

Organs (lung, heart, spleen, liver, and kidney) and skin tissues were collected from the mice on day 7 , day 15 , and day 30 after the PEG-DA-CuS NPs-based PTT treatment to observe histological changes in mice. These organs were embedded in paraffin after immobilization in $4 \%$ paraformaldehyde at $4{ }^{\circ} \mathrm{C}$ for 4 hours. At last, the sections were stained with $\mathrm{H} \& \mathrm{E}$ and representative images were collected using a light microscope.

\section{Hematology and biochemical assay}

Hematology and biochemical assays were conducted by collecting mouse blood on day 7 , day 15 , and day 30 after the PEG-DA-CuS NPs-based PTT treatment or from untreated normal mice. In order to assess the biocompatibility and in vivo toxicity of PEG-DA-CuS NPs, we analyzed kidney function and spleen function by monitoring blood urea nitrogen (BUN), platelet (PLT) level, and urea creatinine (CRE). ALT, total protein, and total bilirubin level were determined to study serological liver function. In addition, blood CRE and BUN were used to detect kidney function, and the PLT level was correlated with spleen function. Neutrophils, red blood cells, monocytes, lymphocytes, and white blood cells were counted by flow cytometry assay, and levels of hemoglobin were tested to evaluate the potential cytotoxic effect and immune response. 


\section{Results and discussion Characterization of PEG-DA-CuS NPs}

The morphology of the as-obtained PEG-DA-CuS NPs was depicted by TEM as indicated in Figure 1A. From the micrograph, we could find that the as-acquired PEG-DA-CuS NPs have the morphology of homogeneously dispersed sphere and a mean diameter of about $10 \mathrm{~nm}$. UV-Vis spectra (Figure 1B) of PEG-DA-CuS displayed a strong absorption peak at $1,065 \mathrm{~nm}$, suggesting the superior potency of PEG-DA-CuS
NPs for converting the absorbed optical energy into heat. After a 10-minute irradiation by 1,064 $\mathrm{nm}$ laser, there was no apparent absorption change in the UV spectra of PEG-DA$\mathrm{CuS}$ NPs, indicating the wonderful photothermal stability. The profile of temperature elevation was investigated and is recorded in Figure 1C. Aqueous solution $(1 \mathrm{~mL})$ containing $1 \mathrm{mg}$ PEG-DA-CuS NPs was put in a quartz cell and subjected to laser irradiation $\left(1,064 \mathrm{~nm}, 1 \mathrm{~W} / \mathrm{cm}^{2}\right)$. A thermographic camera was used to track the increasing
A
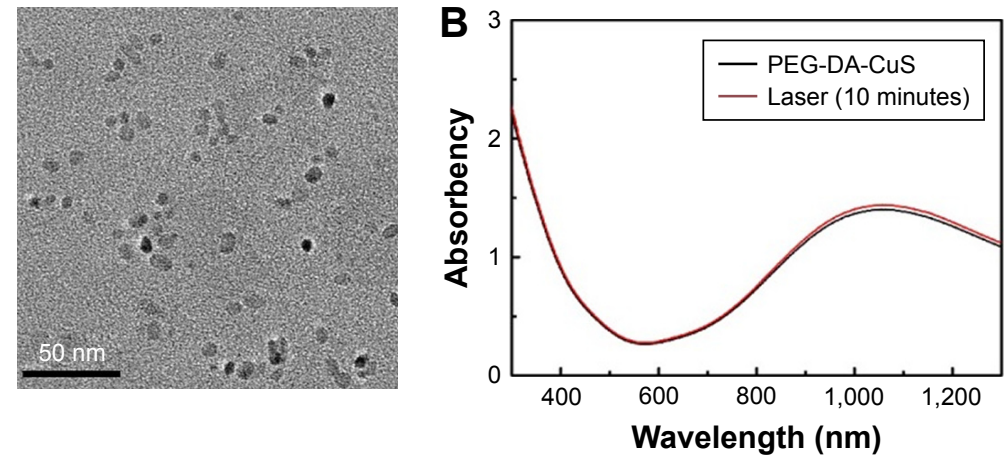

D

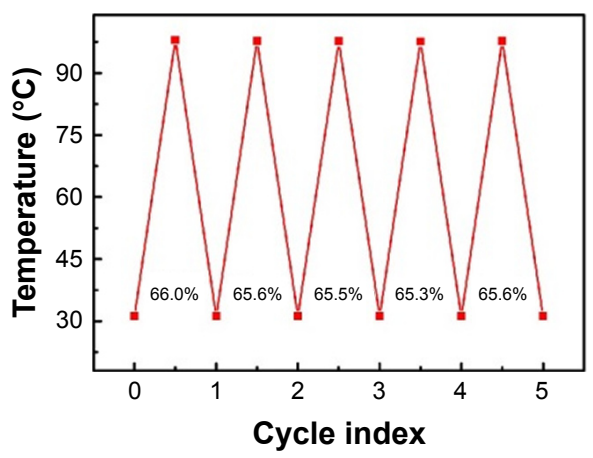

E

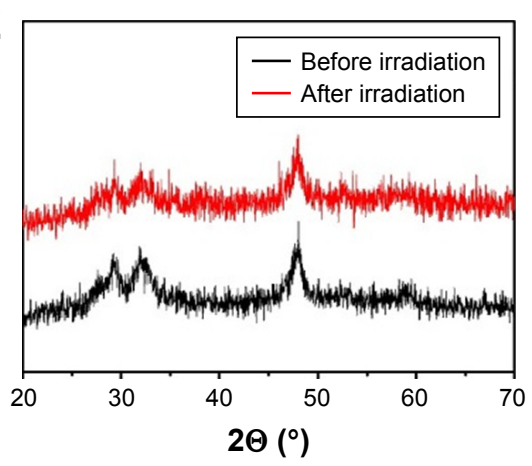

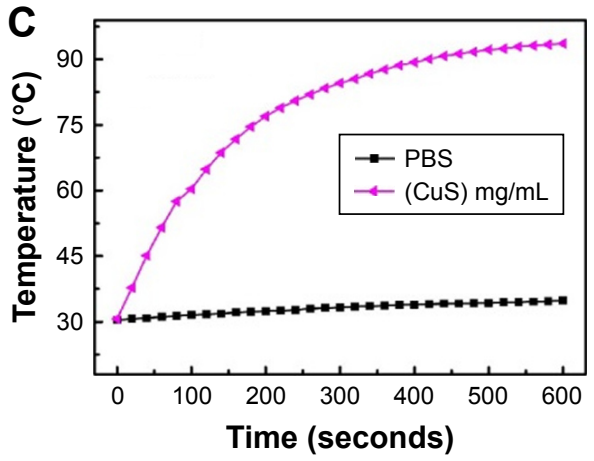

$\mathbf{F}$

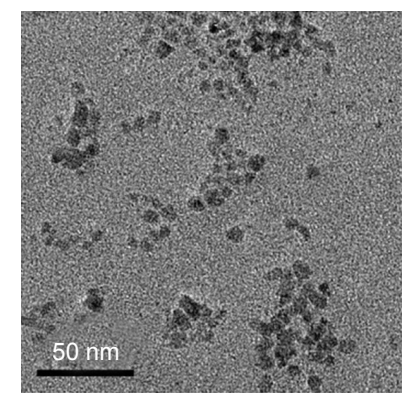

G

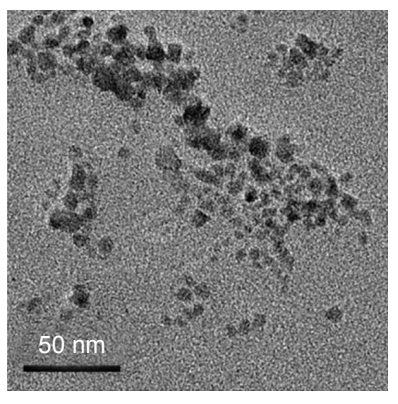

H

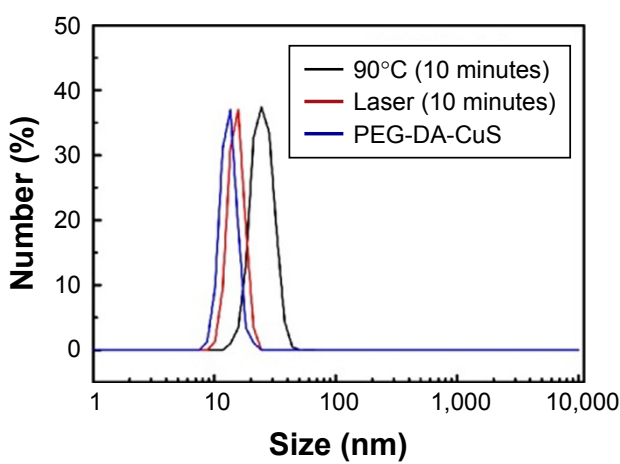

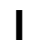

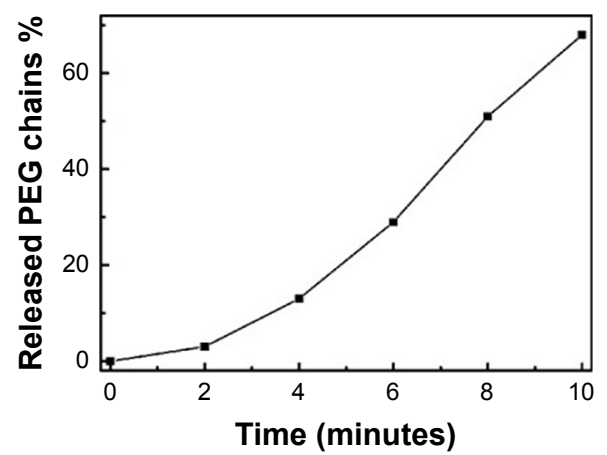

Figure I Characterization of PEG-DA-CuS NPs.

Notes: (A) TEM image of PEG-DA-CuS NPs. (B) UV-Vis spectrum of PEG-DA-CuS NPs before and after NIR laser irradiation (I W/cm², I,064 nm laser, I0 minutes). (C) Temperature-time curve of PBS and PEG-DA-CuS NPs (I mg/mL) dissolved in distilled water. (D) Temperature of PEG-DA-CuS NPs solution (I.43 g, I.4 mL) after five cycles of NIR laser irradiation (I W/cm², I,064 nm laser, 10 minutes)/cooling down (I hour) process. (E) XRD of PEG-DA-CuS NPs before and after NIR laser irradiation (I W/cm², I,064 nm laser, 10 minutes). (F) TEM image of PEG-DA-CuS NPs after NIR laser irradiation (I W/cm², I,064 nm laser, I0 minutes). (G) TEM image of PEG-DACuS NPs after oil bath heating $\left(90^{\circ} \mathrm{C}, 10\right.$ minutes). (H) Particle size distribution of PEG-DA-CuS NPs before NIR laser irradiation, after NIR laser irradiation (I W/cm ${ }^{2}$, I,064 nm laser, 10 minutes), and after oil bath heating $\left(90^{\circ} \mathrm{C}, 10\right.$ minutes). (I) Release of PEG chains from the PEG-DA-CuS after irradiation with I,064 $\mathrm{nm}$ laser (I W/cm $\left.{ }^{2}\right)$ for 10 minutes.

Abbreviations: NIR, near infrared; NP, nanoparticle; PEG, polyethylene glycol; TEM, transmission electron microscopy; UV, ultraviolet; XRD, X-ray powder diffraction. 
temperature. As indicated in Figure 1C, the temperature curve of PEG-DA-CuS NPs solution exhibited significant growth (increased from $30.2^{\circ} \mathrm{C}$ to $91.5^{\circ} \mathrm{C}$ ) 10 minutes after $1,064 \mathrm{~nm}$ laser irradiation; on the contrary, the control solution only increased from $30.2^{\circ} \mathrm{C}$ to $32^{\circ} \mathrm{C}$. These data prove that the PEG-DA-CuS NPs featured such impressive photothermal properties that ensured the success of retro Diels-Alder reaction. According to Figure $1 \mathrm{D}$, the conversion efficiency was estimated to be around $65.6 \%$, and this value did not decrease significantly after repeated laser irradiation, which confirmed that PEG-DA-CuS NPs represented excellent stability as photothermal agents. X-ray power diffraction measurements of PEG-DA-CuS NPs (Figure 1E) exhibited typical diffraction peaks of CuS (JCPDS no 06-0464). There was no detectable change in these NPs after irradiation with 1,064 nm laser, again showing the stability of PEG-DA-CuS NPs. Since the temperature of PEG-DA-CuS NPs when exposed to $1,064 \mathrm{~nm}$ laser for 10 minutes was high enough to induce retro Diels-Alder reaction and release the PEG chain, we next investigated the morphology change and size change of PEG-DA-CuS NPs after laser irradiation via TEM and dynamic light scattering. In Figure $1 \mathrm{~F}-\mathrm{H}$, we could
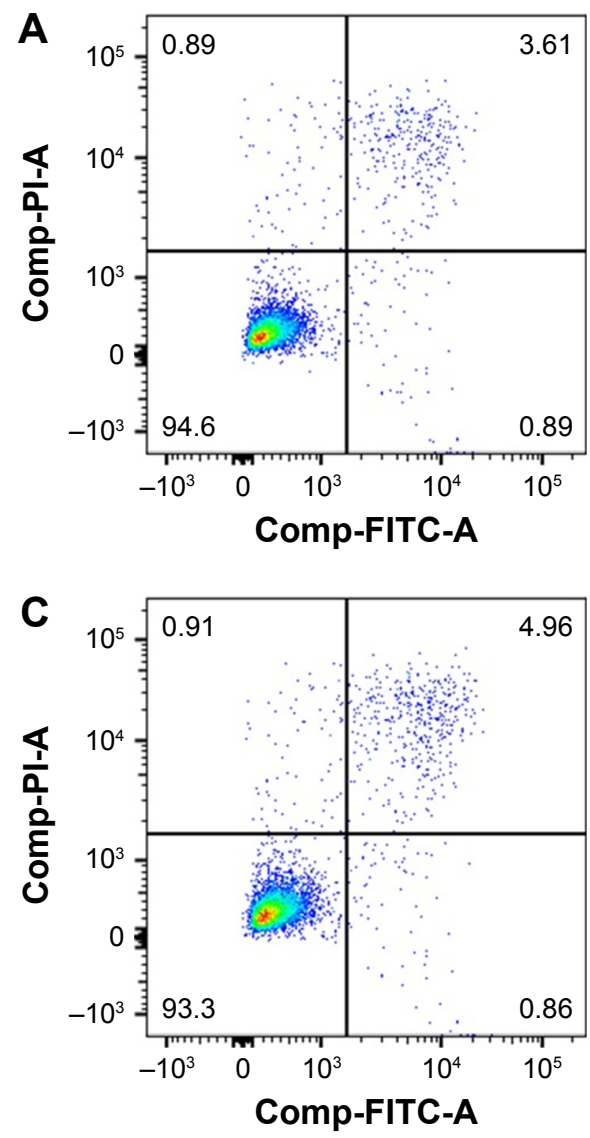

see the aggregate morphology and enhanced hydrodynamic diameter regardless of 10 minutes irradiation with $1,064 \mathrm{~nm}$ laser or heating using oil at $90^{\circ} \mathrm{C}$. All these taken together, we conclude that the as-obtained PEG-DA-CuS NPs had two major properties. On one hand, they maintained good photothermal and structural stability. On the other hand, their superior photothermal effect made it possible that the PEG chain would be released from PEG-DA-CuS NPs once the retro Diels-Alder reaction was triggered by $1,064 \mathrm{~nm}$ laser irradiation. Next, we conducted the iodine-barium assay and analyzed the quantified release of PEG chains from the PEG-DA-CuS NPs. In Figure 1I, it could be seen that little PEG chain was released in the first 5 minutes owing to the low temperature. However, as the temperature rose, more and more PEG was released and $\sim 68 \%$ of the modified $P E G$ chain was detected in the PEG-DA-CuS solution after irradiation with $1,064 \mathrm{~nm}$ laser $\left(1 \mathrm{~W} / \mathrm{cm}^{2}\right)$ for 10 minutes.

\section{In vitro cytotoxicity}

The in vitro cytotoxicity of PEG-DA-CuS NPs was assessed in normal HUVECs via flow cytometry analysis. As displayed in Figure $2 \mathrm{~A}-\mathrm{C}$, no obvious loss of cell viability $(<7 \%)$ existed
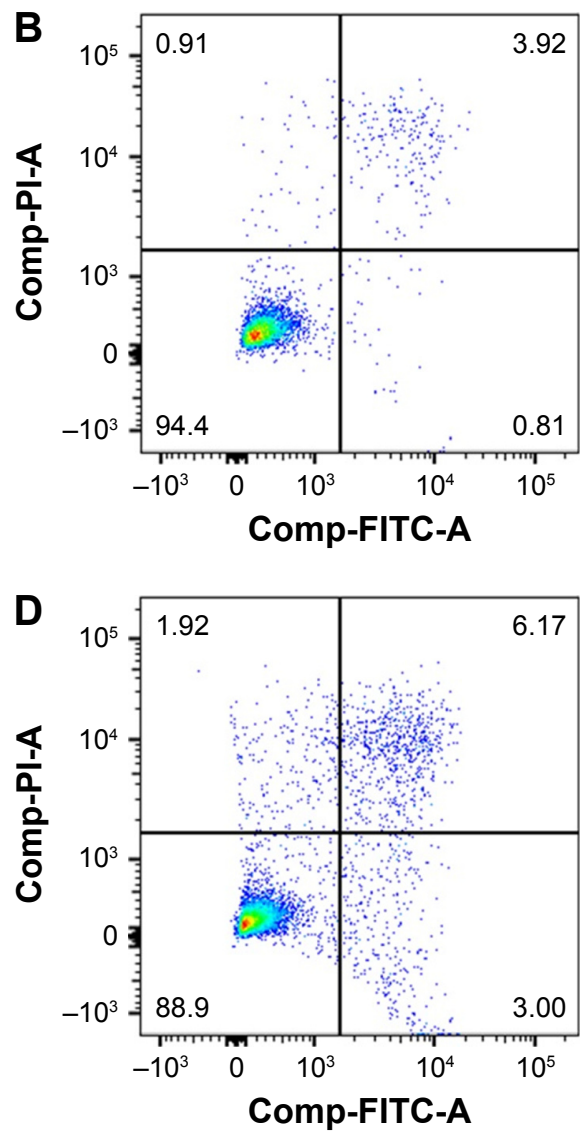

Figure 2 Flow cytometry analysis of HUVECs cultured with PEG-DA-CuS NPs at various concentrations: (A) $0.1 \mathrm{mg} / \mathrm{mL}$; (B) $0.2 \mathrm{mg} / \mathrm{mL}$; (C) $0.4 \mathrm{mg} / \mathrm{mL}$; and (D) I mg/mL. Abbreviations: FITC, fluorescein isothiocyanate; HUVECs, human umbilical vein endothelial cells; NPs, nanoparticles; PEG, polyethylene glycol; PI, propidium iodide. 
when the concentration was below $0.5 \mathrm{mg} / \mathrm{mL}$, indicating the cells demonstrate excellent tolerance to PEG-DA-CuS NPs of low concentration. In addition, even if the drug concentration was raised to $1 \mathrm{mg} / \mathrm{mL}$ (Figure 2D), over $88 \%$ viability could still be reached. These data are good proof of the compatibility of PEG-DA-CuS NPs for practical applications.

\section{Biodistribution of PEG-DA-CuS NPs}

PEG was reported to have the function of prolonging the blood circulation of NPs, and we analyzed the blood retention profiles of PEG-DA-CuS NPs postinjection. As displayed in Figure 3A, the half-life of PEG-DA-CuS NPs in blood is 252 minutes, which proves the long circulation time. As such, we speculated that the removal of PEG from PEG-DA-CuS NPs at the tumor site would shorten the blood circulation of $\mathrm{CuS}$ NPs and further cause the aggregation of $\mathrm{CuS}$ around the tumor, leading to the passive enrichment of $\mathrm{CuS}$ in the tumor. To demonstrate this hypothesis, the biodistribution of CuS NPs was monitored on HeLa-xenograft-bearing mice treated with 10-minute 1,064 nm laser at the power of
$1 \mathrm{~W} / \mathrm{m}^{2}$ at 0.5 hour after the iv injection or with nothing after the injection. We noticed that, as a result of the aggregation of $\mathrm{CuS}$ around the tumor (Figure $3 \mathrm{~B}$ ), a large proportion of the iv-injected CuS NPs was enriched in the tumor tissue through the enhanced permeability and retention effect. By contrast, only a small amount of the CuS NPs was enriched in the tumor due to the long blood circulation time of PEG (Figure 3C).

\section{In vitro antitumor efficacy}

Live/dead double-staining method (Figure 4) was applied to verify the therapeutic outcome of PTT in cells in vitro under different treatments. Calcein-AM stained live cells with green fluorescence, while EthD-1 showed dead cells with red fluorescence. No red color could be detected in the control group (HeLa cells treated with nothing) and two negative groups (HeLa cells irradiated by 1,064 nm laser (10 minutes, $1 \mathrm{~W} / \mathrm{cm}^{2}$ ) or incubated with PEG-DA-CuS NPs alone), indicating negligible cytotoxic damage to the HeLa cells was exerted by these treatments. On the contrary, a mass
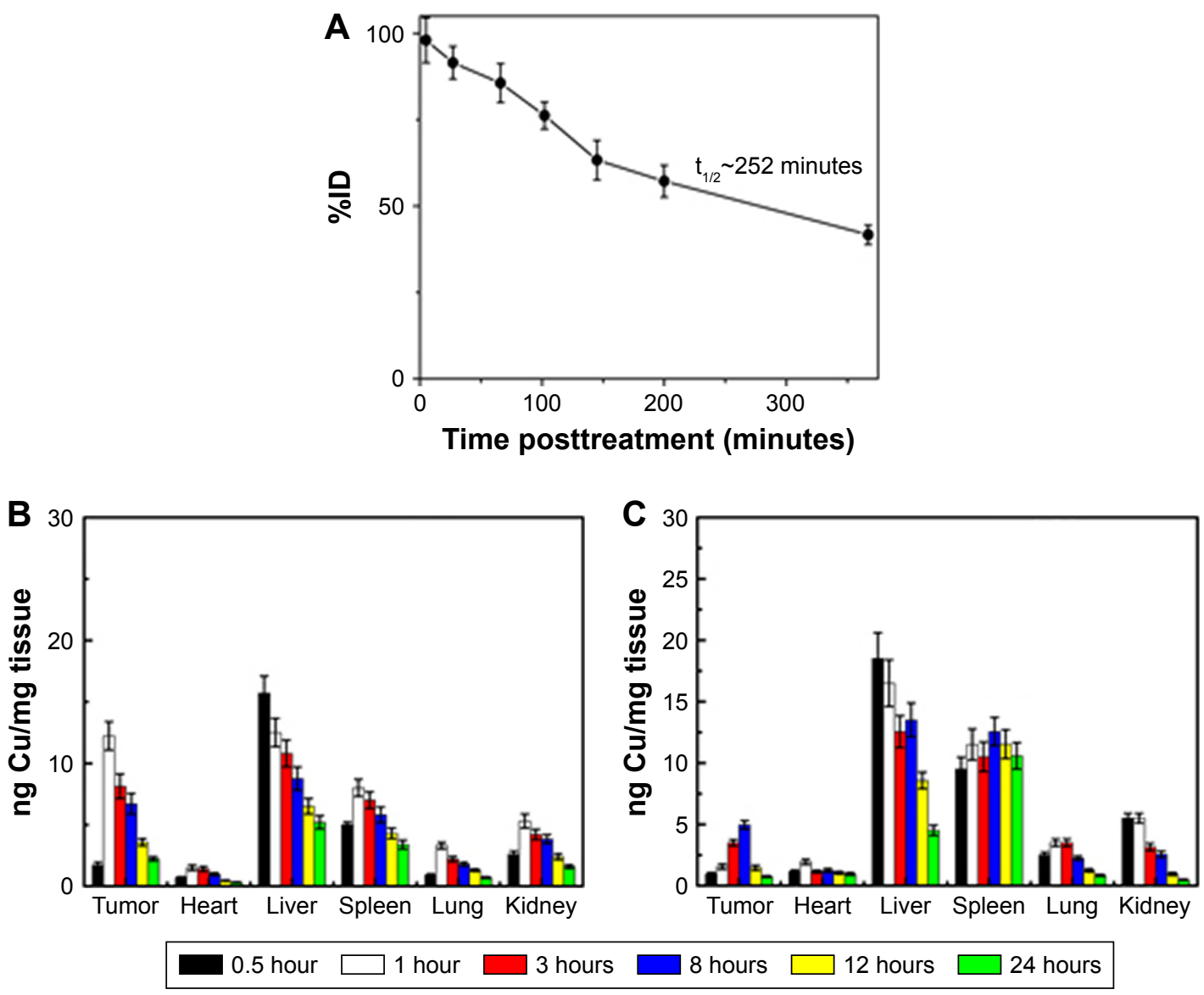

Figure 3 (A) In vitro distribution profiles of CuS NPs in blood. (B) Biodistribution analysis of CuS NPs with laser irradiation (I W/cm², I,064 nm laser, I0 minutes). (C) Biodistribution analysis of CuS NPs without laser irradiation.

Abbreviation: NP, nanoparticle. 

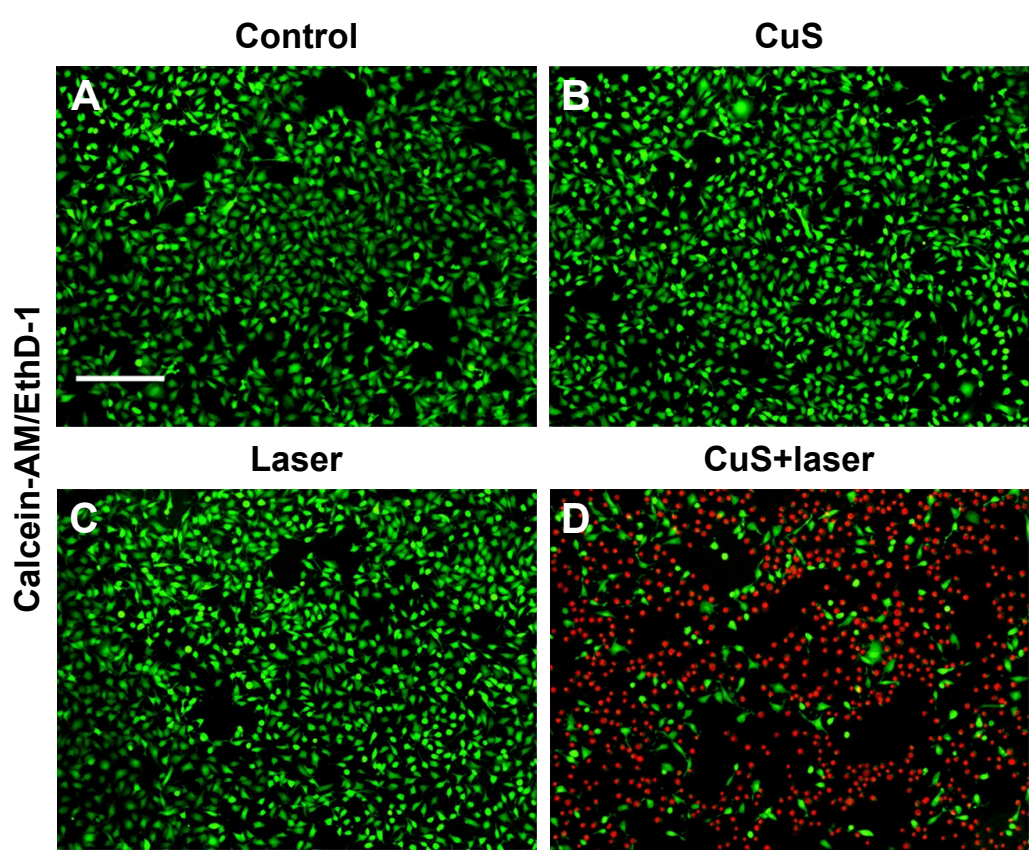

Figure 4 Fluorescence images of HeLa cells.

Notes: (A) The cells were incubated with nothing. (B) The cells were incubated with PEG-DA-CuS NPs $(200 \mu \mathrm{g} / \mathrm{mL})$ alone. (C) The cells were exposed to laser irradiation (I W/cm², I,064 nm laser, 10 minutes) alone. (D) The cells were incubated with PEG-DA-CuS NPs (200 $\mu \mathrm{g} / \mathrm{mL})$ and exposed to laser irradiation (I W/cm², I,064 nm laser, 10 minutes). Calcein-AM (green fluorescence representing live cells) and EthD-I (red fluorescence representing dead cells) were used as indicators. The scale bar is $50 \mu \mathrm{m}$. Magnification is $10 X$

Abbreviations: AM, acetoxymethyl; EthD-I, ethidium homodimer-I; NPs, nanoparticles; PEG, polyethylene glycol.

of red fluorescence was observed when the cells received the PTT treatment rendered by PEG-DA-CuS NPs, which confirmed the wonderful therapeutic efficacy of PEG-DACuS NPs against HeLa cells.

\section{In vivo antitumor efficacy}

Subsequently, the in vivo antitumor efficacy of PTT rendered by PEG-DA-CuS NPs on mice bearing HeLa tumors was investigated. As represented in Figure 5A, the tumor from the control group (saline group) and other two negative groups (PEG-DA-CuS NPs alone and 1,064 nm laser alone) showed faster growth rates, accompanied by an $\sim 7$-fold increase in volume (from day 12 to day 0 ). On the contrary, the PEG-DA$\mathrm{CuS}$ NPs treatment significantly ablated the tumor volume. Compared to day 0 , the tumor volume of the PEG-DA-CuS NPs plus laser group reached 1.2-fold on day 12, showing dramatic photothermal effect in the elimination of cancer simultaneously. According to the recorded body weight curve of mice depicted in Figure 5B, no abnormal weight changes were detected throughout the whole process, confirming the PEG-DA-CuS NPs-based PTT would not cause apparent acute toxicity in mice bearing HeLa tumor.

$\mathrm{H} \&$ E staining (Figure 5C) and Ki67 staining (Figure 5D) were used to evaluate the therapeutic effect after different treatments. Tumor tissues from mice treated with PTT rendered by PEG-DA-CuS NPs presented remarkable necrosis and apoptosis. Therefore, we concluded that the PTT rendered by PEG-DA-CuS NPs offered an excellent therapeutic efficacy in cancer treatment.

\section{Safety evaluation}

Major organs (heart, lung, spleen, kidney, and liver) of mice receiving PTT rendered by PEG-DA-CuS NPs were collected and weighed to assess the in vivo cytotoxicity. Compared with the control group, H\&E staining pictures obtained from the experimental group displayed in Figure 6A represented negligible histological abnormalities, necrosis, inflammation lesions, and pulmonary fibrosis, demonstrating excellent in vivo biocompatibility of PEG-DA-CuS NPs.

We also selected four nude mice and picked the skin tissues in the same location of the mice body to conduct safety evaluation via histological analysis. The selected skin tissues were treated in different ways (treated with nothing as the control group, injected with PEG-DA-CuS NPs alone, laser irradiation alone, and injected with PEG-DA-CuS NPs and irradiated with $1,064 \mathrm{~nm}$ laser), and the $\mathrm{H} \& \mathrm{E}$ results are shown in Figure 6B. Compared with the control group, the skin tissues of mice treated with PEG-DA-CuS NPs alone or laser irradiation alone represented no apparent histological changes. On the contrary, the skin structure of those receiving PEG-DA-CuS NPs treatment under laser irradiation was destroyed, leaving only two to three layers of stratum corneum. 


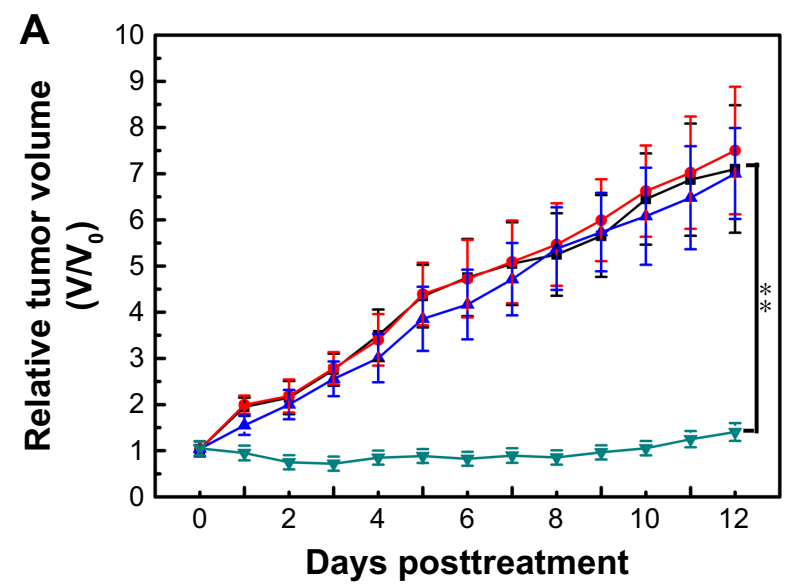

A
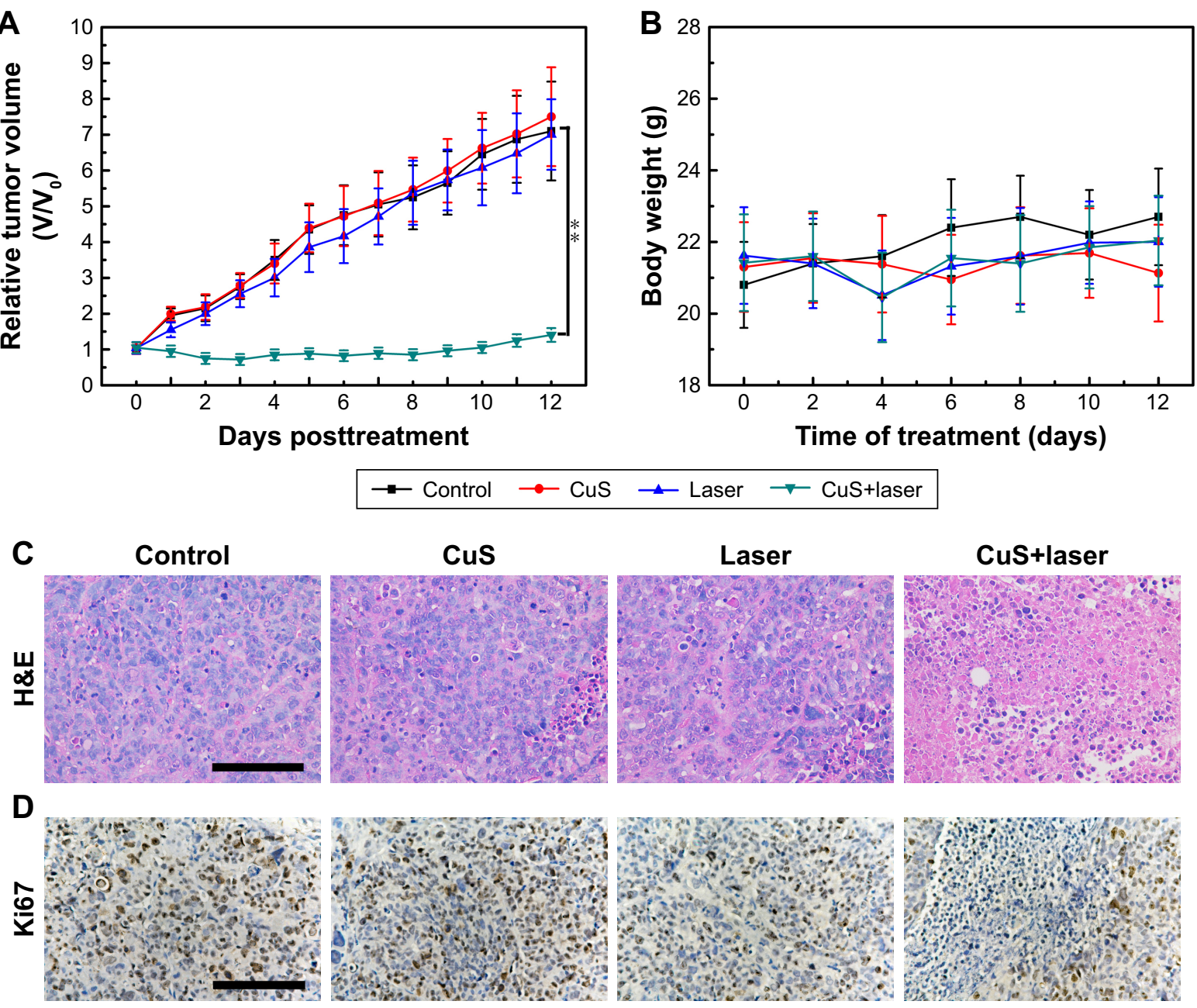

Figure 5 (A) Tumor growth curves of HeLa tumor-bearing mice subjected to different treatments as displayed. The data are expressed as the mean $\pm S D$ ( $n=8$ ). $* * P<0.01$. (B) Body weight of HeLa tumor-bearing mice subjected to different treatments. (C) IHC staining images of HeLa tumor sections from mice subjected to different treatments, obtained with H\&E staining. (D) Representative IHC for Ki67 staining images of HeLa tumor sections obtained from mice subjected to different treatments. The scale bar is $50 \mu \mathrm{m}$. Abbreviation: IHC, immunohistochemistry.

These data proved that damages on the normal tissues occurred only in NP-enriched location with the help of laser irradiation. Therefore, guiding the laser to the lesions (such as tumors) directly without penetrating the normal tissues will be a solution. We believe, with the development of modern medical techniques, approaches to direct the laser to the lesions (such as tumors) without penetrating the skin will be discovered. In addition, serum biochemical study and hematology assay were conducted to further investigate the potential cytotoxicity of PEG-DA-CuS NPs. No obvious changes in statistical significance were found in total bilirubin, ALT, total protein, CRE, BUN, and PLT when compared with the control group (untreated mice), indicating liver, kidney, and spleen functions were normal and their metabolism was not disrupted after PEG-DA-CuS NPs treatment (Figure 7A and B). The clinical fate of NPs was decided by hepatotoxicity. As shown in Figure $7 \mathrm{C}$, hematopoietic and aerobic capacity reflected by the counts of hemoglobin, white blood cells, and red blood cells was similar compared with the control group (untreated mice). There were no detectable changes in peripheral blood monocytes, lymphocytes, and neutrophil counts (Figure 7D) when compared with the control group, suggesting no potential immune responses occurred throughout the treatment. Taken together, all these data offer solid evidence that PEG-DA-CuS NPs have promising clinical applications.

\section{Conclusion}

We reported a kind of ideal photothermal agent, PEG-DA-CuS NPs, for NIR laser-induced PTT. Success of this methodology relies on the occurrence of retro Diels-Alder reaction triggered by the intrinsic photothermal property of CuS NPs, allowing the aggregation of photothermal agents in the tumor. In addition, based on the results of in vitro experiments, we noticed that PEG-DA-CuS NPs show excellent photothermal stability 

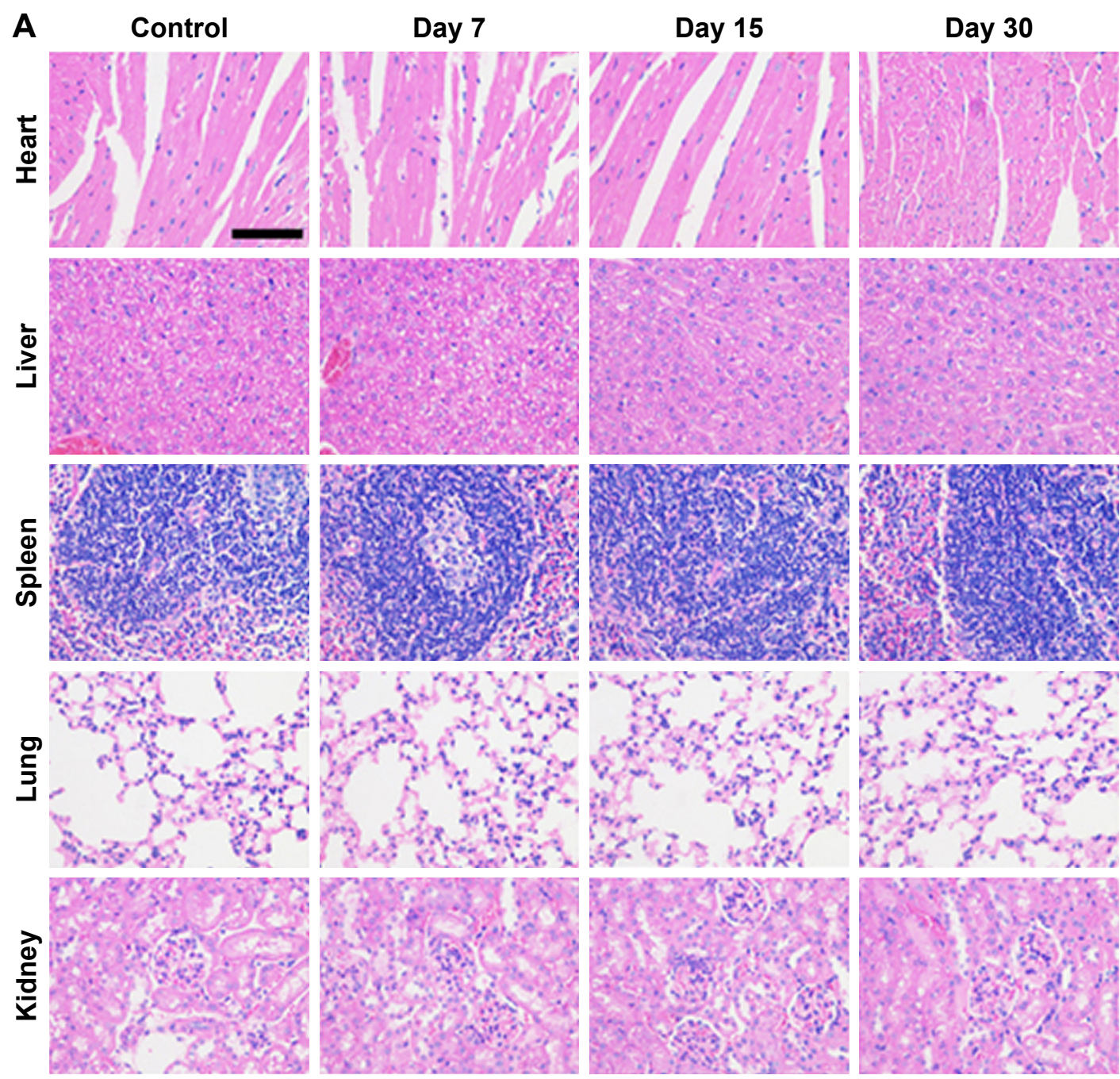

B
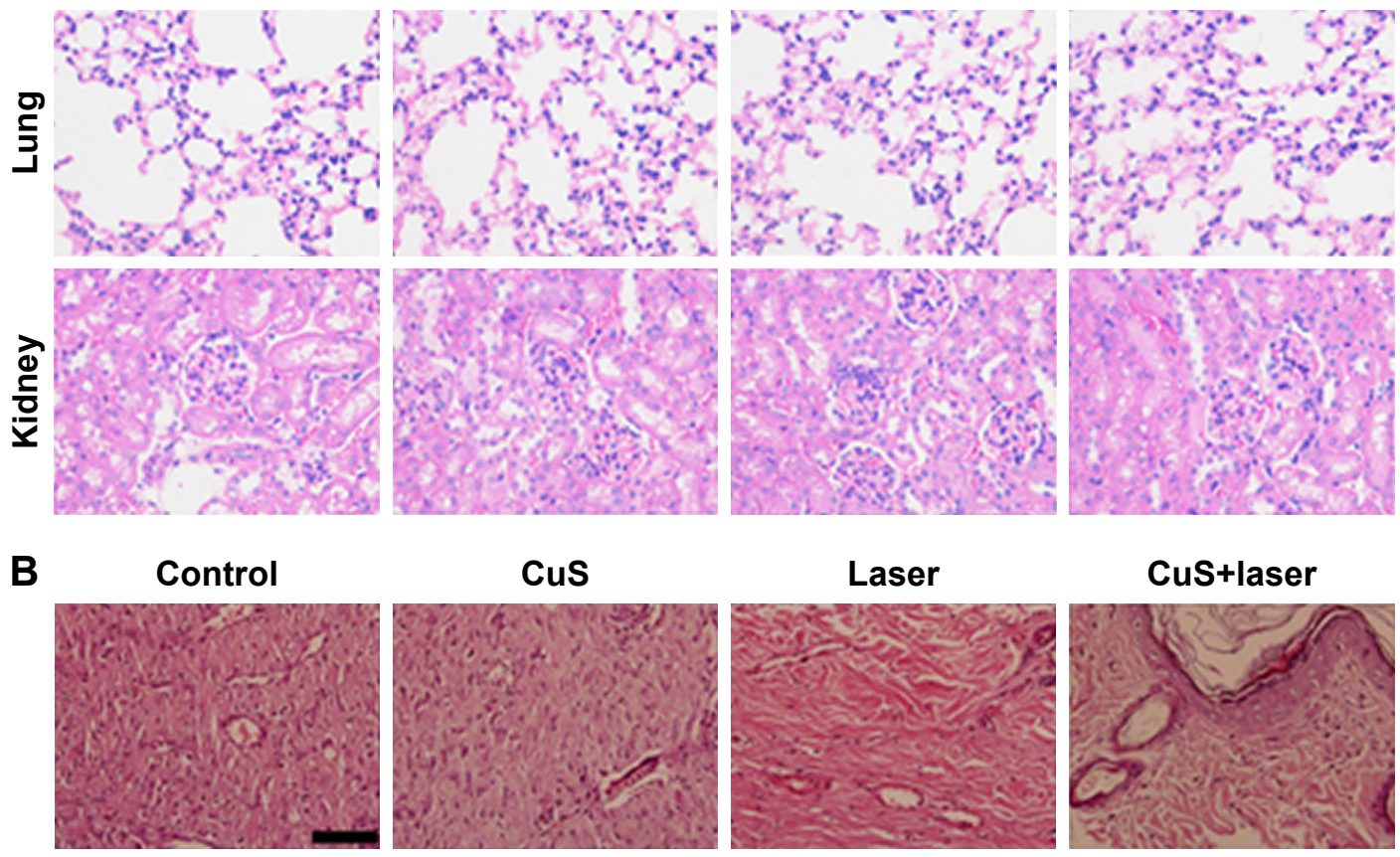

Figure 6 (A) Histological images of the HE-stained heart, liver, spleen, lung, and kidney harvested from mice after treatment with PTT rendered by PEG-DA-CuS NPs or from mice treated with nothing as the control group. (B) Histological images of the H\&E-stained skin harvested from mice after different treatments (treated with nothing as the control group, injected with PEG-DA-CuS NPs alone, laser irradiation alone, and injected with PEG-DA-CuS NPs and irradiated with I,064 nm laser). We selected four nude mice and picked the skin tissues in the same location of the mice body to conduct the histological analysis. The scale bar is $50 \mu \mathrm{m}$.

Abbreviations: NPs, nanoparticles; PEG, polyethylene glycol; PTT, photothermal therapy.

and high heat conversion efficiency. Moreover, the in vivo experiments indicated the tumor size of mice receiving PTT rendered by PEG-DA-CuS NPs was significantly reduced, prolonging the lives of mice, and further confirmed that PEGDA-CuS NPs exhibited low cytotoxicity. Therefore, we are quite sure that PEG-DA-CuS NPs can be used as promising photothermal agents in the ablation of tumors.

\section{Acknowledgment}

This study was supported by the National Natural science Foundation of China (nos. 21764015, 11464047, 31360613), Autonomous Region Youth Science and Technology Innovation Personnel Training Project (QN2016YX0504), and University Level Key Project of Yili Normal University (2016WNZD02). 

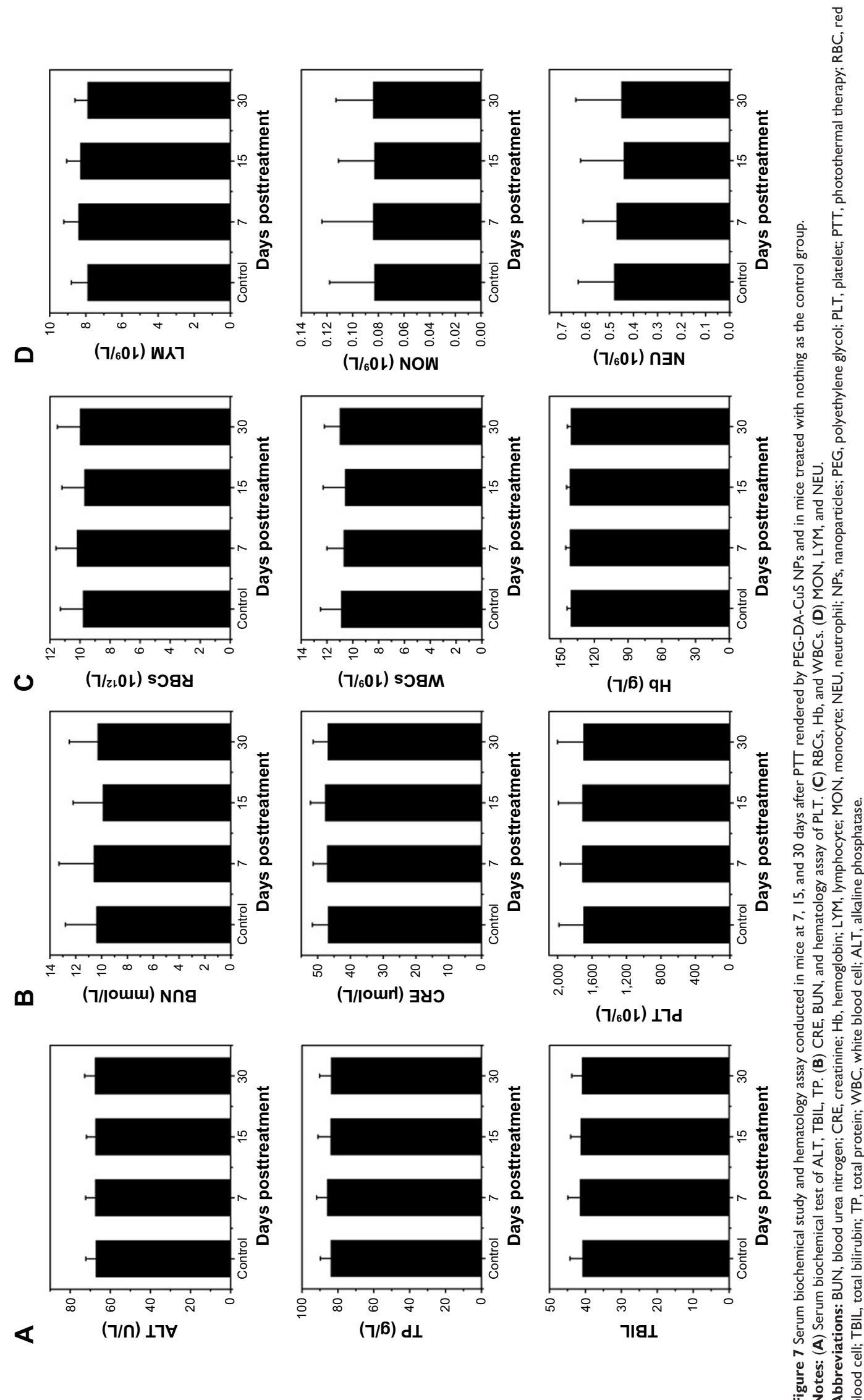

$(7 / 6) \mathrm{qH}$
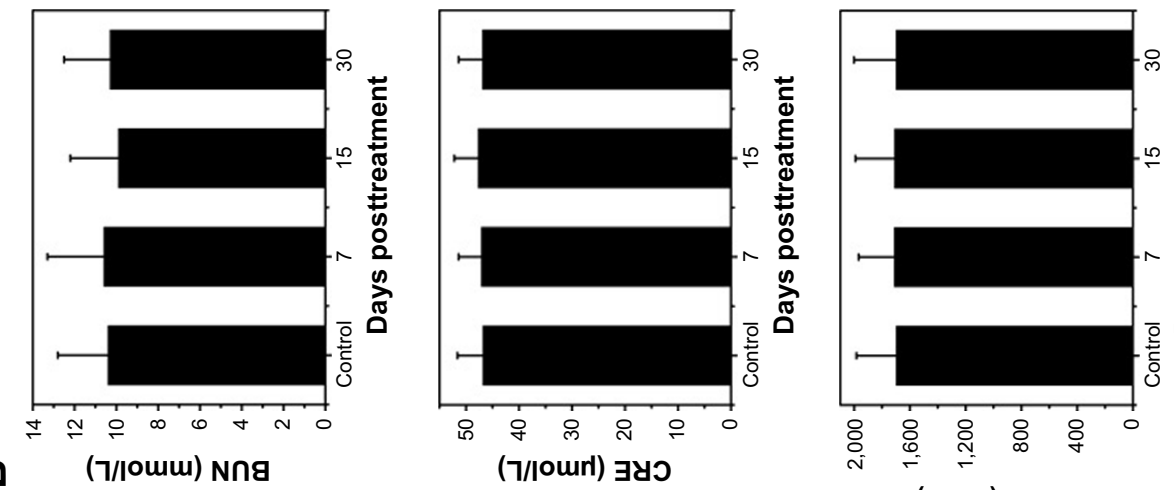

$(7 / 60 \mathrm{l}) \perp 7 d$
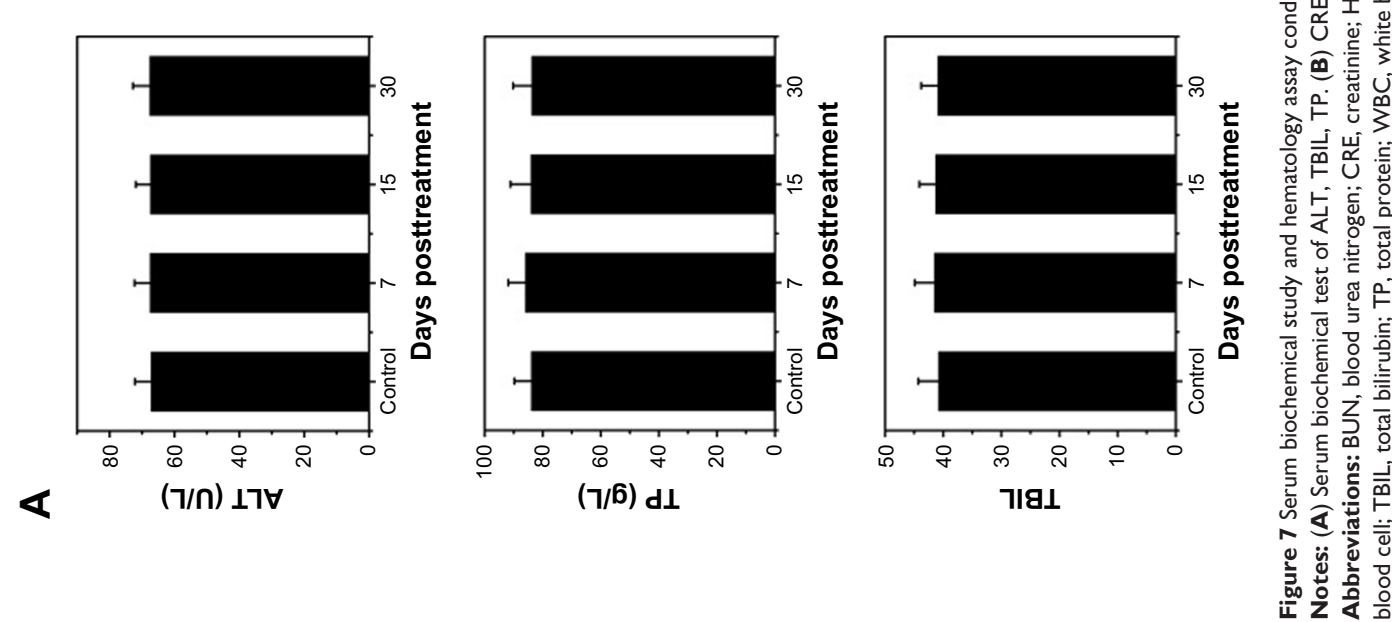


\section{Author contributions}

All authors contributed toward data analysis, drafting and revising the paper and agree to be accountable for all aspects of the work.

\section{Disclosure}

The authors report no conflicts of interest in this work.

\section{References}

1. Joyce JA, Pollard JW. Microenvironmental regulation of metastasis. Nat Rev Cancer. 2009;9(4):239-252.

2. Quail DF, Joyce JA. Microenvironmental regulation of tumor progression and metastasis. Nat Med. 2013;19(11):1423-1437.

3. Mathew R, Karantza-Wadsworth V, White E. Role of autophagy in cancer. Nat Rev Cancer. 2007;7(12):961-967.

4. Folkman J. Angiogenesis and apoptosis. Semin Cancer Biol. 2003;13(2): 159-167.

5. Wang K, Wu X, Wang J, Huang J. Cancer stem cell theory: therapeutic implications for nanomedicine. Int J Nanomedicine. 2013;8: 899p-908p.

6. Dearnaley DP, Khoo VS, Norman AR, et al. Comparison of radiation side-effects of conformal and conventional radiotherapy in prostate cancer: A randomised trial. Lancet. 1999;353(9149):267-272.

7. Lecaros RL, Huang L, Lee TC, Hsu YC. Nanoparticle Delivered VEGF-A siRNA Enhances Photodynamic Therapy for Head and Neck Cancer Treatment. Mol Ther. 2016;24(1):106-116.

8. Zhang C, Cheng X, Chen M, et al. Fluorescence guided photothermal/ photodynamic ablation of tumours using $\mathrm{pH}$-responsive chlorin e6-conjugated gold nanorods. Colloids Surf B Biointerfaces. 2017;160: $345-354$.

9. Taratula O, Patel M, Schumann C, et al. Phthalocyanine-loaded graphene nanoplatform for imaging-guided combinatorial phototherapy. Int J Nanomedicine. 2015;10:2347-2362.

10. Zhang C, Ren J, Hua J, et al. Multifunctional $\mathrm{Bi}_{2} \mathrm{WO}_{6}$ Nanoparticles for CT-Guided Photothermal and Oxygen-free Photodynamic Therapy. ACS Appl Mater Interfaces. 2018;10(1):1132-1146.

11. Hou L, Yang X, Ren J, et al. A novel redox-sensitive system based on single-walled carbon nanotubes for chemo-photothermal therapy and magnetic resonance imaging. Int J Nanomedicine. 2016;11:607-624.

12. Millenbaugh NJ, Baskin JB, Desilva MN, Elliott WR, Glickman RD. Photothermal killing of Staphylococcus aureus using antibody-targeted gold nanoparticles. Int J Nanomedicine. 2015;10:1953-1960.

13. Liu Z, Liu J, Wang R, du Y, Ren J, Qu X. An efficient nano-based theranostic system for multi-modal imaging-guided photothermal sterilization in gastrointestinal tract. Biomaterials. 2015;56:206-218.

14. Fay BL, Melamed JR, Day ES. Nanoshell-mediated photothermal therapy can enhance chemotherapy in inflammatory breast cancer cells. Int J Nanomedicine. 2015;10:6931-6941.

15. Xi J, da L, Yang C, et al. Mn²+-coordinated PDA@DOX/PLGA nanoparticles as a smart theranostic agent for synergistic chemo-photothermal tumor therapy. Int J Nanomedicine. 2017;12:3331-3345.
16. Yang RM, Fu CP, Fang JZ, et al. Hyaluronan-modified superparamagnetic iron oxide nanoparticles for bimodal breast cancer imaging and photothermal therapy. Int J Nanomedicine. 2017;12:197-206.

17. Thapa RK, Choi JY, Poudel BK, Choi HG, Yong CS, Kim JO. Receptortargeted, drug-loaded, functionalized graphene oxides for chemotherapy and photothermal therapy. Int J Nanomedicine. 2016;11:2799-2813.

18. Zhao P, Ren S, Liu Y, Huang W, Zhang C, He J. PL-W ${ }_{18} \mathrm{O}_{49}-\mathrm{TPZ}$ Nanoparticles for Simultaneous Hypoxia-Activated Chemotherapy and Photothermal Therapy. ACS Appl Mater Interfaces. 2018;10(4): 3405-3413.

19. Zhang C, Ren J, Yang Y, et al. Ultra-sensitive diagnosis of orthotopic patient derived hepatocellular carcinoma by Fe@graphene nanoparticles in MRI. RSC Adv. 2016;6(115):113919-113923.

20. Ren S, Cheng X, Chen M, et al. Hypotoxic and Rapidly Metabolic PEGPCL-C3-ICG Nanoparticles for Fluorescence-Guided Photothermal/ Photodynamic Therapy against OSCC. ACS Appl Mater Interfaces. 2017; 9(37):31509-31518.

21. Cantu T, Walsh K, Pattani VP, et al. Conductive polymer-based nanoparticles for laser-mediated photothermal ablation of cancer: synthesis, characterization, and in vitro evaluation. Int J Nanomedicine. 2017;12: 615-632.

22. Wang Z, Chen Z, Liu Z, et al. A multi-stimuli responsive gold nanocagehyaluronic platform for targeted photothermal and chemotherapy. Biomaterials. 2014;35(36):9678-9688.

23. Madani SY, Shabani F, Dwek MV, Seifalian AM. Conjugation of quantum dots on carbon nanotubes for medical diagnosis and treatment. Int J Nanomedicine. 2013;8:941-950.

24. Dong K, Liu Z, Liu J, et al. Biocompatible and high-performance amino acids-capped MnWO4 nanocasting as a novel non-lanthanide contrast agent for X-ray computed tomography and T(1)-weighted magnetic resonance imaging. Nanoscale. 2014;6(4):2211-2217.

25. Liu Z, Liu X, Ran X, Ju E, Ren J, Qu X. Single-layer tungsten oxide as intelligent photo-responsive nanoagents for permanent male sterilization. Biomaterials. 2015;69:56-64.

26. Liu Z, Liu J, Wang R, du Y, Ren J, Qu X. An efficient nano-based theranostic system for multi-modal imaging-guided photothermal sterilization in gastrointestinal tract. Biomaterials. 2015;56:206-218.

27. $\mathrm{Mu} J$, Meng X, Chen L, et al. Highly stable and biocompatible $\mathrm{W}_{18} \mathrm{O}_{49}$ (a)PEG-PCL hybrid nanospheres combining CT imaging and cancer photothermal therapy. RSC Adv. 2017;7(18):10692-10699.

28. Meng Z, Wei F, Wang R, et al. NIR-Laser-Switched In Vivo Smart Nanocapsules for Synergic Photothermal and Chemotherapy of Tumors. Adv Mater. 2016;28(2):245-253.

29. Zhang L, Gao S, Zhang F, Yang K, Ma Q, Zhu L. Activatable hyaluronic acid nanoparticle as a theranostic agent for optical/photoacoustic imageguided photothermal therapy. ACS Nano. 2014;8(12):12250-12258.

30. Yamashita S, Fukushima H, Niidome Y, Mori T, Katayama Y, Niidome T. Controlled-release system mediated by a retro Diels-Alder reaction induced by the photothermal effect of gold nanorods. Langmuir. 2011; 27(23):14621-14626.
International Journal of Nanomedicine

\section{Publish your work in this journal}

The International Journal of Nanomedicine is an international, peerreviewed journal focusing on the application of nanotechnology in diagnostics, therapeutics, and drug delivery systems throughout the biomedical field. This journal is indexed on PubMed Central, MedLine, CAS, SciSearch $®$, Current Contents ${ }^{\circledR} /$ Clinical Medicine,
Dovepress

Journal Citation Reports/Science Edition, EMBase, Scopus and the Elsevier Bibliographic databases. The manuscript management system is completely online and includes a very quick and fair peer-review system, which is all easy to use. Visit http://www.dovepress.com/ testimonials.php to read real quotes from published authors. 